\title{
Transaortic transcatheter aortic valve implantation: Results of the Polish arm of the ROUTE registry
}

\author{
Dariusz Jagielak ${ }^{1}$, Peter Bramlage ${ }^{2}$, Rafał Pawlaczyk ${ }^{1}$, Maciej Brzeziński ${ }^{1}$, \\ Marcin Fijałkowski ${ }^{1}$, Grzegorz Łaskawski ${ }^{1}$, Dariusz Ciećwierz ${ }^{3}$, \\ Jan Rogowski ${ }^{4}$, Radosław Kozaryn ${ }^{1}$ \\ ${ }^{1}$ Department of Cardiovascular Surgery, Medical University of Gdansk, Gdansk, Poland \\ ${ }^{2}$ Institute for Pharmacology and Preventive Medicine, Berlin, Germany \\ ${ }^{3}$ Department of Cardiology, Medical University of Gdansk, Gdansk, Poland \\ ${ }^{4}$ Department of Cardiac Surgery, Medical University of Gdansk, Gdansk, Poland
}

\begin{abstract}
Background: Transaortic (TAo) transcatheter aortic valve implantation (TAVI) is an alternative approach in patients considered to be at high risk for classical open surgery with poor peripheral vessel access. The purpose of this study was to determine the feasibility of using TAo access for TAVI procedures employing the Edwards SAPIEN transcatheter heart valve. The primary objective was to determine overall 30-day mortality.

Methods: A total of 32 patients with severe aortic valve stenosis underwent TAo-TAVI using Edwards SAPIEN bioprostheses. Postoperative results were collected according to the Registry of the Utilization Of the TAo-TAVI approach using the Edwards SAPIEN Valve (ROUTE) study protocol. Complications were assessed using Valve Academic Research Consortium2 (VARC-2) criteria.
\end{abstract}

Results: The mean age of the population was $80.9 \pm 5.2$ years, with $53.1 \%$ being female. All patients received either the SAPIEN XT or the SAPIEN 3 bioprosthesis (Edwards Lifesciences). Device success was achieved in 100\% of cases. One (3.25\%) patient subsequently suffered an aortic dissection and required ascending aorta replacement. Paravalvular leakage was absent or mild in 26 (81\%) patients, and moderate in 6 (19\%) patients. Other complications included permanent pacemaker implantation in 2 (6.5\%), and transient post operative delirium in 2 (6.5\%) patients. The total hospital stay was $6.7 \pm 2.4$ days. New York Heart Association class decreased significantly on follow-up. Thirty-day mortality rate was 2 (6.5\%) patients.

Conclusions: Use of TAo access for TAVI procedures has a reasonable clinical outcome and is a safe alternative to the transfemoral and transapical approaches, especially for patients with high-risk peripheral vessel access. (Cardiol J 2015; 22, 6: 651-656)

Key words: aortic stenosis, transaortic approach, transcatheter aortic valve implantation, mini sternotomy, ROUTE registry

Address for correspondence: Radosław Kozaryn, MD, Department of Cardiac Surgery, Medical University of Gdansk, ul. Dębinki 7, 80-952 Gdańsk, Poland, e-mail: radekkozaryn@gmail.com

Received: 27.04.2015 Accepted: 03.07.2015 


\section{Introduction}

The development of the transcatheter aortic valve implantation (TAVI) procedure by Cribier et al. [1] has provided a suitable alternative to open vale replacement surgery for elderly high risk patients with severe aortic stenosis [2]. Since its introduction, TAVI has been predominantly performed using transfemoral (TF) access [3]. However, this approach disqualifies patients with severe peripheral atherosclerosis or physiological abnormalities of the vasculature $[4,5]$. Alternatively, a transapical approach (TA-TAVI) can be employed [6]. Still, this route has certain limitations; it is not recommended in patients with chronic lung diseases or poor left ventricular function, and is associated with a risk of serious bleeding complications [7-9]. The use of transaortic (TAo) access is a more recently developed alternative to TA-TAVI and TF-TAVI $[10,11]$. This involves insertion of the catheter via an upper reversed L ministernotomy, which is a safe and well-established procedure in classic cardiac surgery, followed by valve implantation through the ascending aorta. This approach has been reported to provide ample visibility for the surgeon and fast recovery for most patients [12]. To date, there have been encouraging preliminary results published with regards to TAo-TAVI, however more detailed information is required to assess the true efficacy and safety of the procedure [10]. The material presented herein is taken from the Polish arm of the Registry Of the Utilization of the TAo-TAVI approach using the Edwards SAPIEN Valve (ROUTE). This was an international, multi-center, prospective, observational registry carried out in 25 sites across Europe [13].

\section{Methods}

\section{Patient selection}

From May 2013 to June 2014, 32 consecutive patients who were assigned by the local Heart Team to undergo TAo-TAVI with an Edwards SAPIEN XT or SAPIEN 3 transcatheter heart valve (THV) were enrolled into the registry. Members of the Heart Team included a cardiac surgeon, an interventional cardiologist, and a cardiologist. All study patients were previously disqualified from open heart surgery. Severe stenosis was defined as an aortic valve surface area less than $1.0 \mathrm{~cm}^{2}$ and a mean transaortic gradient $>40 \mathrm{~mm} \mathrm{Hg}$, as determined using echocardiography examination. Prior to the intervention, all patients underwent a complete work-up, comprising physical exami- nation, New York Heart Association (NYHA) assessment, and baseline laboratory testing. Aortic annulus diameter, topography, and morphology of the ascending aorta were assessed exclusively by computed tomography angiography in all patients. Exclusion criteria were: congenital unicuspid/bicuspid aortic valves; presence of any cardiac mass, vegetation, or suspicion of endocarditis; intolerance of anticoagulation or antiplatelet therapy; and excessive calcification of the aorta at the planned access point. Postoperative results were collected according to the ROUTE study protocol, and complications were assessed using the Valve Academic Research Consortium-2 (VARC-2) criteria [14].

\section{TAo-TAVI procedure}

All procedures were performed by the Heart Team in a hybrid operating room with an accessible heart-lung machine. General anesthesia was used in all cases. A pacing wire was first introduced through the right jugular vein into the right ventricle. A reversed $\mathrm{L}$ ministernotomy was performed down to the second or third intercostal space, then the ascending aorta was exposed and directly palpated to find the optimal cannulation site. The selected location should be free of calcification and at least $6 \mathrm{~cm}$ from the aortic annulus. Thereafter, two 4-0 Prolene purse-string sutures were placed. A bolus of intravenous heparin $(1.5 \mathrm{mg} / \mathrm{kg})$ was administered to obtain an activated clotting time of at least $250 \mathrm{~s}$. A diagnostic catheter was then introduced through the right radial artery and placed under fluoroscopic guidance in the non-coronary aortic sinus. Fluoroscopy was performed to confirm the correct puncture site and its position with respect to the annulus. The aortic wall was subsequently punctured with a needle to allow introduction of a $6 \mathrm{~F}$ sheath. Following this, a $6 \mathrm{~F}$ multipurpose catheter (MPA, Boston Scientific) was placed and the valve was crossed with a soft-tip straight wire. The soft wire was changed for a pre-shaped extra stiff wire placed in the apex of the left ventricle. The $6 \mathrm{~F}$ sheath was subsequently changed for an ASCENDRA 2 delivery system (Edwards Lifesciences, Irvine, CA; $22 \mathrm{~F}$ sheath for 23 - and $26-\mathrm{mm}$ valves and $26 \mathrm{~F}$ for $29-\mathrm{mm}$ valve). Pre-dilatation with a balloon was performed under rapid pacing. Finally, the valve was introduced through the delivery system after careful positioning in the annulus. The position of the prosthesis was confirmed using a dose of contrast medium. The valve was deployed under rapid pacing using a 2 -step inflation technique, with a second shot of contrast to ensure an accurate final 
position. After implantation, a further bolus of contrast was given to assess the position of the THV and allow simultaneous estimation of paravalvular leakage (PVL) and coronary status. The decision of whether to carry out post-dilatation was made after estimating the shape of the valve and after quantification of PVL.

\section{Echocardiography}

Each patient underwent transesophageal echocardiography (TEE) assessment at least 3 times throughout their hospital course: on admission; post-procedurally to estimate PVL, contractility of the left ventricle, and to assess pericardial effusion; and at discharge.

\section{Follow-up, complications, and mortality}

A 30-day follow-up was conducted in an outpatient clinic setting. Events occurring within the $1^{\text {st }}$ month were considered to procedure-related and as such, were documented. Complication rates were recorded according to the VARC-2 criteria. Thirty-day mortality was defined as all-cause mortality during hospitalization or within 30 days of admission.

\section{Statistical analyses}

Statistical analyses were performed using the statistical suite StatSoft, Inc. (2011; STATISTICA data analysis software system version 10.0) and Microsoft Excel. Quantitative variables are given as the arithmetic mean and standard deviation, or median with range and 95\% confidence interval (CI). Qualitative variables are presented as absolute values and percentages.

\section{Results}

\section{Population characteristics}

The study population consisted of 32 patients, of which 17 were female (53.1\%; Table 1$)$. The mean age was $80.9 \pm 5.2$ years, with a mean height of $164.6 \pm 11.1 \mathrm{~cm}$, and mean weight of $74.7 \pm$ $\pm 16.0 \mathrm{~kg}$. The EuroSCORE range was $1-11 \%$ (mean $2.84 \%$ ) with mean Society Thoracic Surgeons score of $8.7 \%$ (range 2-19\%). A small proportion of patients suffered moderate mitral valve insufficiency (9.4\%), while $59.4 \%$ suffered mild, and $31.3 \%$ suffered none. Comorbidities within the population were frequent, with hypertension being the most prevalent at $87.5 \%$. The other most common conditions were coronary artery disease $(59.4 \%)$, diabetes mellitus (15.6\%), myocardial infarction (12.5\%), and atrial fibrillation (12.5\%).
Table 1. Patient characteristics ( $n=32$ ).

\begin{tabular}{lc}
\hline Age [years] & $80.9 \pm 5.2$ \\
Female gender & $17(53.1 \%)$ \\
Height [cm] & $164.6 \pm 11.1$ \\
Weight [kg] & $74.7 \pm 16.0$ \\
Mitral insufficiency: & \\
None & $10(31.3 \%)$ \\
Mild & $19(59.4 \%)$ \\
Moderate & $3(9.4 \%)$ \\
Comorbidities: & \\
Coronary artery disease & $19(59.4 \%)$ \\
Myocardial infarction & $4(12.5 \%)$ \\
PCl (prior 6 weeks) & $1(3.1 \%)$ \\
Atrial fibrillation & $4(12.5 \%)$ \\
Hypertension & $28(87.5 \%)$ \\
Diabetes mellitus & $5(15.6 \%)$ \\
Stroke & $2(6.2 \%)$ \\
COPD & $3(9.4 \%)$ \\
\hline
\end{tabular}

$\mathrm{PCl}$ - percutaneous coronary intervention; COPD — chronic obstructive pulmonary disease

Table 2. Echocardiographic findings $(n=32)$.

\begin{tabular}{lc}
\hline Ejection fraction [\%] & $57.9 \pm 9.0$ \\
Peak PG [mm Hg] & $78.9 \pm 23.3$ \\
Mean PG [mm Hg] & $48.3 \pm 14.4$ \\
$\mathrm{~V}_{\max }[\mathrm{m} / \mathrm{s}]$ & $4.3 \pm 0.6$ \\
\hline PG - pressure gradient; $\mathrm{V}_{\max }$ - maximal velocity
\end{tabular}

Patients who were also suitable for other access routes were also included in the study, with $\mathrm{TF}$ access judged to be possible for $87.5 \%$ of them.

\section{Echocardiographic findings prior to the intervention}

Prior to the TAo-TAVI procedure, the mean ejection fraction was measured to be $57.9 \pm 9.0 \%$ (Table 2). The peak pressure gradient was $78.9 \pm$ $\pm 23.3 \mathrm{~mm} \mathrm{Hg}$, with a mean pressure gradient of $48.3 \pm 14.4 \mathrm{~mm} \mathrm{Hg}$. The mean maximal velocity $\left(\mathrm{V}_{\text {max }}\right)$ of the population was $4.3 \pm 0.6 \mathrm{~m} / \mathrm{s}$.

\section{Procedural characteristics}

The majority of patients underwent implantation of the SAPIEN XT THV (93.8\%), with the remaining procedures being carried out using the more recently approved SAPIEN 3 (7.2\%; Table 3). The mean duration of the procedure, defined as the time from the first skin incision until completion of the final suture, was $96.7 \pm 34.8 \mathrm{~min}$. The mean 
Table 3. Procedural characteristics $(n=32)$.

\begin{tabular}{lc}
\hline Valve type used: & \\
SAPIEN XT & $30(93.8 \%)$ \\
SAPIEN 3 & $2(7.2 \%)$ \\
Valve size: & \\
$23 \mathrm{~mm}$ & $11(34 \%)$ \\
$26 \mathrm{~mm}$ & $8(25 \%)$ \\
$29 \mathrm{~mm}$ & $13(40 \%)$ \\
Skin-to-skin time $[\mathrm{min}]$ & $96.7(34.8 \%)$ \\
Volume of contrast $[\mathrm{mL}]$ & $119.9 \pm 35.7$ \\
Fluoroscopy time $[\mathrm{min}]$ & $14.7 \pm 7.1$ \\
Post-dilatation & $8(25.0 \%)$ \\
\hline
\end{tabular}

Table 4. Procedural outcomes $(n=32)$.

\begin{tabular}{lc}
\hline Paravalvular leakage: & \\
None to trace & $11(34 \%)$ \\
Mild & $15(47 \%)$ \\
Moderate & $6(19 \%)$ \\
Severe & $0(0.0 \%)$ \\
Creatinine level [mg/dL] & $1.5(1.3)$ \\
Atrioventricular block & $2(6.5 \%)$ \\
Pacemaker implantation & $2(6.5 \%)$ \\
Aortic dissection & $1(3.1 \%)$ \\
Transient post operative delirium & $2(6.5 \%)$ \\
Hospital stay [days] & $6.7 \pm 2.4$ \\
Discharged home & $19(59.4 \%)$ \\
30-day mortality & $2(6.5 \%)$ \\
\hline
\end{tabular}

volume of contrast agent used was $119.9 \pm 35.7 \mathrm{~mL}$, and the mean fluoroscopy time was $14.7 \pm 7.1 \mathrm{~min}$, which included positioning of the pacing wires. Post-dilatation was carried out in $8(25 \%)$ cases.

\section{Follow-up}

Paravalvular leakage was classified as none to trace in 11 (34\%) patients, mild in 15 (47\%), and moderate in $6(19 \%)$ patients. The mean peak pressure gradient was significantly reduced from $78.9 \pm 23.3 \mathrm{~mm} \mathrm{Hg}$ before TAVI to $9.4 \pm 2.9 \mathrm{~mm} \mathrm{Hg}$ after, with the mean $\mathrm{V}_{\max }$ decreasing from $4.3 \pm$ $\pm 0.6 \mathrm{~m} / \mathrm{s}$ to $2.1 \pm 0.3 \mathrm{~m} / \mathrm{s}$. After the procedure, the mean creatinine level was found to be $1.5 \pm 1.3 \mathrm{mg} / \mathrm{dL}$ (Table 4). Permanent atrioventricular (AV) block occurred in $2(6.5 \%)$ cases, with both requiring peacemaker implantation. The mean duration of hospital stay was $6.7 \pm 2.4$ days, with the majority of patients $(59.4 \%)$ being discharged home. Two

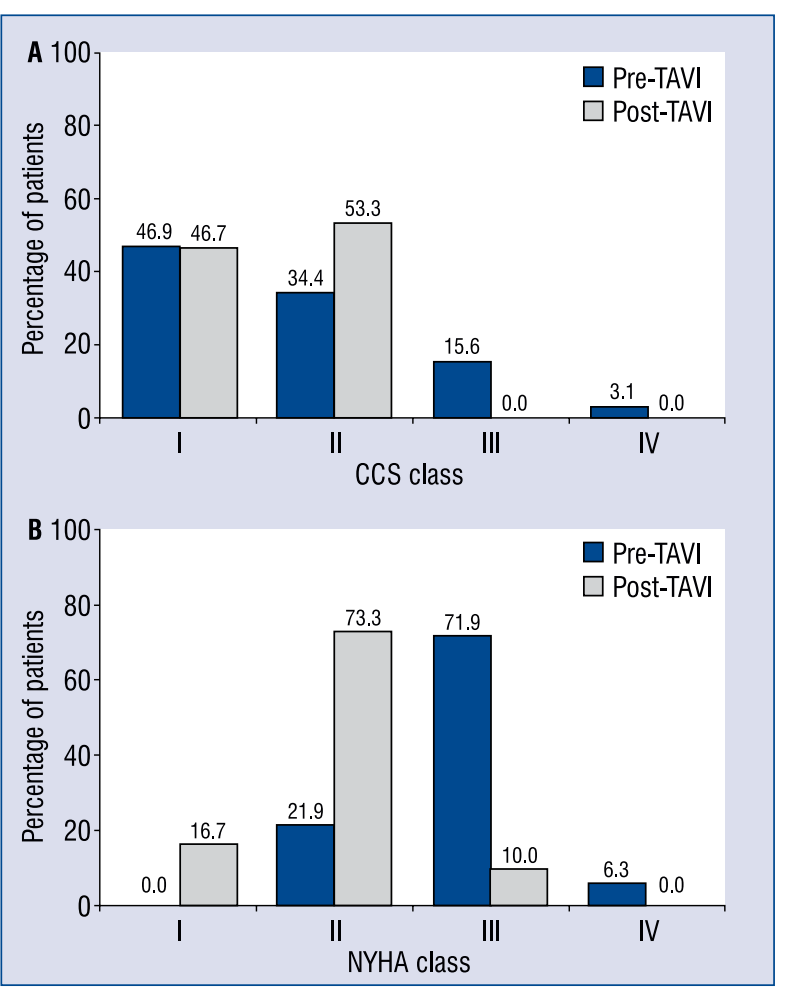

Figure 1. Changes in grading of angina pectoris and heart failure; A. Canadian Cardiovascular Society (CCS); B. New York Heart Association (NYHA); TAVI - transcatheter aortic valve implantation.

(6.5\%) patients died during the 30-day follow-up period, both due to respiratory complications. Quality of life improvement was best evidenced by significant decreases in the proportion of patients with angina pectoris at Canadian Cardiovascular Society class III/IV and heart failure at NYHA class III/IV (Fig. 1).

\section{Discussion}

The direct transaortic approach for TAVI is a more recently developed alternative to the use of $\mathrm{TA}$ access in patients for whom the $\mathrm{TF}$ route is contraindicated [10]. In early reports, Clarke et al. [11] found good ease of access and visibility using this method, while Lardizabal et al. [15] demonstrated superior mortality rates compared to the TA approach, with comparable complication rates. The short distance from the puncture site to the annulus enabled by the TAo access provides excellent and immediate tactile feedback [16]. This short delivery system is easy to navigate and gives consistent confidence in accurate prosthesis positioning [17]. Furthermore, it avoids the aortic 
arch, and is therefore less likely to be associated with neurological complications caused by calcific debris, as is sometimes seen with the TF approach. Injury to the subvalvular apparatus of the mitral valve, ventricular septal defects, and development of a false aneurysm of the apex of the left ventricle are all well-described complications of the TA approach that are not associated with TAo-TAVI [6]. Furthermore, major bleeding occurs infrequently in the latter; however, should such a serious complication occur intraoperatively, the ministernotomy may be easily converted to full sternotomy, providing instant access to the aortic root [5].

In our series, any PVL after the TAo-TAVI procedure was regarded as trace or mild, with a small proportion of patients experiencing that classed as moderate. There were no cases of severe leakage, which is similar to previously reported results $[5,18,19]$. This demonstrates the accurate valve positioning that is possible using this procedure.

In terms of complications, 1 patient suffered from aortic dissection, which was diagnosed immediately after valve deployment. A successful ascending aorta replacement was performed without removal of the otherwise well-positioned SAPIEN prosthesis. We observed no major strokes in our group of patients and only 2 patients suffered from transient ischemia. The most prevalent complication was the development of AV block with a requirement for pacemaker implantation, which is in agreement with previous studies regarding TAo-TAVI [20, 21]. In contrast, for the TA approach, this complication has been shown to occur less frequently [21-23], indicating that positioning of the THV may be deeper when the TAo route is used [24, 25].

All-cause mortality during the 30-day followup period was similar to that reported for other TAo-TAVI studies $[18,20]$. Furthermore, it was significantly lower than values documented for TA-TAVI $[3,15,22]$.

\section{Limitations of the study}

Whilst these data demonstrate the potential of TAo-TAVI as an alternative to TF and TA, there were a number of limitations. Firstly, all patients were operated on in the same center by the same Heart Team. Secondly, owing to the small number of SAPIEN 3 valves employed, it was not possible to directly compare the outcomes between these and the SAPIEN XT.

\section{Conclusions}

The use of TAo access for TAVI procedures provides a satisfactory clinical outcome and is a safe alternative for patients with poor peripheral vessel access and common comorbidities. This study shows the TAo approach to be a very promising prospective for the future. Our findings provide some insight into the further developments and investigation required in this growing area.

\section{Conflict of interest: None declared}

\section{References}

1. Cribier A, Eltchaninoff H, Bash A et al. Percutaneous transcatheter implantation of an aortic valve prosthesis for calcific aortic stenosis: First human case description. Circulation, 2002; 106: 3006-3008.

2. Kuck KH, Hamm C. Executive summary of the position paper of the German Cardiac Society on quality criteria for the implementation of transcatheter aortic valve implantation (TAVI. Eur Heart J, 2015; 36: 328-330.

3. Thomas M, Schymik G, Walther T et al. Thirty-day results of the SAPIEN aortic Bioprosthesis European Outcome (SOURCE) Registry: A European registry of transcatheter aortic valve implantation using the Edwards SAPIEN valve. Circulation, 2010; 122: 62-69. doi: 10.1161/CIRCULATIONAHA.109.907402.

4. Latsios G, Gerckens U, Grube E. Transaortic transcatheter aortic valve implantation: a novel approach for the truly "no-access option" patients. Catheter Cardiovasc Interv, 2010; 75: 1129-1136. doi: 10.1002/ccd.22378.

5. Thourani VH, Li C, Devireddy C et al. High-risk patients with inoperative aortic stenosis: use of transapical, transaortic, and transcarotid techniques. Ann Thoracic Surgery, 2015; 99: 817-825. doi: 10.1016/j.athoracsur.2014.10.012.

6. Wendler $\mathrm{O}$, Walther T, Nataf P et al. Trans-apical aortic valve implantation: univariate and multivariate analyses of the early results from the SOURCE registry. Eur J Cardio-thoracic Surgery, 2010; 38: 119-127. doi: 10.1016/j.ejcts.2009.12.048.

7. Pasic M, Buz S, Dreysse $\mathrm{S}$ et al. Transapical aortic valve implantation in 194 patients: problems, complications, and solutions. Ann Thoracic Surgery, 2010; 90: 1463-1469. doi: 10.1016/j.athoracsur.2010.05.072.

8. Al-Attar N, Ghodbane W, Himbert D et al. Unexpected complications of transapical aortic valve implantation. Ann Thoracic Surgery, 2009; 88: 90-94. doi: 10.1016/j.athoracsur.2009.03.070.

9. Bleiziffer S, Ruge H, Mazzitelli D et al. Survival after transapical and transfemoral aortic valve implantation: Talking about two different patient populations. J Thoracic Cardiovasc Surgery, 2009; 138: 1073-1080. doi: 10.1016/j.jtcvs.2009.07.031.

10. Bapat V, Khawaja MZ, Attia R et al. Transaortic transcatheter aortic valve implantation using Edwards Sapien valve: A novel approach. Catheter Cardiovasc Interv, 2012; 79: 733-740. doi: 10.1002/ccd.23276. 
11. Clarke A, Wiemers P, Poon KK et al. Early experience of transaortic TAVI: The future of surgical TAVI? Heart, Lung Circ, 2013; 22: 265-269. doi: 10.1016/j.hlc.2012.11.002.

12. Protopapas AD, Amrani M. Traction suture modification of the Cosgrove hemisternotomy technique. J Heart Valve Disease, 2011; 20: 189-190.

13. Bramlage $\mathrm{P}$, Romano $\mathrm{M}$, Bonaros $\mathrm{N}$ et al. Transaortic transcatheter aortic valve implantation: Rationale and design of the first multicenter, multinational prospective registry (ROUTE). BMC Cardiovasc Disord, 2014; 14: 152-157.

14. Kappetein AP, Head SJ, Genereux P et al. Updated standardized endpoint definitions for transcatheter aortic valve implantation: the Valve Academic Research Consortium-2 consensus document (VARC-2). Eur J Cardio-thorac Surg, 2012; 42: S45-S60. doi: 10.1093/ejcts/ezs533.

15. Lardizabal JA, O'Neill BP, Desai HV et al. The transaortic approach for transcatheter aortic valve replacement: Initial clinical experience in the United States. J Am Coll Cardiol, 2013; 61: 2341-2345. doi: 10.1016/j.jacc.2013.02.076.

16. Ferre GF, Latib A, Maisano F, Naganuma T, Alfieri O, Colombo A. Transaortic valve implantation with the direct flow medical valve in an emergency situation of post-valvuloplasty severe aortic regurgitation. Cardiovasc Revascular Med, 2014; 16: 317-319. doi: 10.1016/j.carrev.2014.12.006.

17. Okuyama K, Jilaihawi H, Mirocha J et al. Alternative access for balloon-expandable transcatheter aortic valve replacement: Comparison of the transaortic approach using right anterior thoracotomy to partial J-sternotomy. J Thoracic Cardiovasc Surg, 2015; 149: 789-797. doi: 10.1016/j.jtcvs.2014.10.062.

18. Hayashida K, Romano M, Lefevre $\mathrm{T}$ et al. The transaortic approach for transcatheter aortic valve implantation: A valid alternative to the transapical access in patients with no peripheral vascular option. A single center experience. Eur J Cardio-thoracic Surg, 2013; 44: 692-700. doi: 10.1093/ejcts/ezt037.

19. Thourani VH, Gunter RL, Neravetla S et al. Use of transaortic, transapical, and transcarotid transcatheter aortic valve replacement in inoperable patients. Ann Thoracic Surg, 2013; 96: 1349_ -1357. doi: 10.1016/j.athoracsur.2013.05.068.

20. Amrane H, Porta F, van Boven AJ et al. Transcatheter aortic valve implantation using a direct aortic approach: A single-centre Heart Team experience. Interactive Cardiovasc Thoracic Surg, 2014; 19: 777-7781. doi: 10.1093/icvts/ivu247.

21. Tanawuttiwat T, O'Neill BP, Cohen MG et al. New-onset atrial fibrillation after aortic valve replacement: Comparison of transfemoral, transapical, transaortic, and surgical approaches. J Am Coll Cardiol, 2014; 63: 1510-1519. doi: 10.1016/j. jacc.2013.11.046.

22. Webb JG, Altwegg L, Boone RH et al. Transcatheter aortic valve implantation: impact on clinical and valve-related outcomes. Circulation, 2009; 119: 3009-3016. doi: 10.1161/CIRCULATIONAHA.108.837807.

23. Eltchaninoff H, Prat A, Gilard M et al. Transcatheter aortic valve implantation: early results of the FRANCE (FRench Aortic National CoreValve and Edwards) registry. Eur Heart J, 2011; 32: 191-197. doi: 10.1093/eurhearti/ehq261.

24. Martinez-Selles M, Bramlage P, Thoenes M, Schymik G. Clinical significance of conduction disturbances after aortic valve intervention: current evidence. Clin Research Cardiol, 2015; 104: 1-12. doi: 10.1007/s00392-014-0739-6.

25. Urena M, Mok M, Serra V et al. Predictive factors and long-term clinical consequences of persistent left bundle branch block following transcatheter aortic valve implantation with a balloon-expandable valve. J Am Coll Cardiol, 2012; 60: 1743-1752. doi: 10.1016/j.jacc.2012.07.035 